\title{
Redundancy or heterogeneity in the electric activity of the biceps brachii muscle? Added value of PCA-processed multi-channel EMG muscle activation estimates in a parallel-fibered muscle
}

\author{
Didier Staudenmann ${ }^{\mathrm{a}, *}$, Dick F. Stegeman ${ }^{\mathrm{b}, \mathrm{c}}$, Jaap H. van Dieën ${ }^{\mathrm{b}}$ \\ ${ }^{a}$ Movement and Sport Science, Department of Medicine, Faculty of Science, University of Fribourg, 1700 Fribourg, Switzerland \\ ${ }^{\mathrm{b}}$ Move Research Institute Amsterdam, Faculty of Human Movement Sciences, VU University Amsterdam, 1081 BT Amsterdam, The Netherlands \\ ${ }^{c}$ Donders Institute for Brain, Cognition E Behavior, Department of Neurology/Clinical Neurophysiology, Radboud University Nijmegen Medical Centre, The Netherlands
}

\begin{abstract}
Conventional bipolar EMG provides imprecise muscle activation estimates due to possibly heterogeneous activity within muscles and due to improper alignment of the electrodes with the muscle fibers. Principal component analysis (PCA), applied on multi-channel monopolar EMG yielded substantial improvements in muscle activation estimates in pennate muscles. We investigated the degree of heterogeneity in muscle activity and the contribution of PCA to muscle activation estimates in biceps brachii (BB), which has a relatively simply parallel-fibered architecture. EMG-based muscle activation estimates were assessed by comparison to elbow flexion forces in isometric, two-state isotonic contractions in eleven healthy male subjects. Monopolar EMG was collected over the entire surface of the BB with about 63 electrodes. Estimation quality of different combinations of EMG channels showed that heterogeneous activation was found mainly in medio-lateral direction, whereas adding channels in the longitudinal direction added largely redundant information. Multi-channel bipolar EMG amplitude improved muscle activation estimates by $5-14 \%$ as compared to a single bipolar. PCA-processed monopolar EMG amplitude yielded a further improvement of (12-22\%). Thus multi-channel EMG, processed with PCA, substantially improves the quality of muscle activation estimates compared conventional bipolar EMG in BB.
\end{abstract}

\section{Introduction}

Ever since the work of Inman and Ralston (1952) muscle activation has been estimated using single bipolar EMG electrode configurations. Although muscle activation is not a physiological variable, it is a commonly used term to represent the combined effect of recruitment and rate coding in a scalar value, ranging from $0 \%$ to $100 \%$. Estimates of muscle activation based on conventional bipolar EMG, however, have limitations (Farina et al., 2004; Staudenmann et al., 2006). Importantly, activity can be heterogeneous within muscles during voluntary contractions, as was shown with multi-channel EMG (Eriksson et al., 1984; Holtermann et al., 2005; Staudenmann et al., 2005, 2009a; van Zuylen et al., 1988, Vieira et al., 2011; Wolf et al., 1998), as well as with ultrasound imaging (Bojson-Moller et al., 2004; Darby et al., 2012; Finni et al., 2003; Pappas et al., 2002). Thus information obtained by a single bipolar

* Corresponding author. Address: Movement and Sport Science, Department of Medicine, University of Fribourg, Bd. de Pérolles 90, CH-1700 Fribourg, Switzerland. Tel.: +41263007286.

E-mail address: didier.staudenmann@unifr.ch (D. Staudenmann).

URL: http://www.unifr.ch/ssm/fr/unite/team/DS (D. Staudenmann).
EMG will not be representative for the entire muscle and consequently muscle activation estimates derived from multi-channel EMG (collecting from different parts of the muscle) are superior to those derived from a single bipolar EMG (Staudenmann et al., 2010).

Another limitation in estimating muscle activity with bipolar EMG is caused by misalignment between the electrode pair and the muscle fiber direction. A bipolar configuration should preferably be aligned to the muscle fiber direction (De Luca, 1997; Farina et al., 2004; Hermens et al., 2000). Otherwise the measured EMG amplitude (Merletti et al., 1999) and the quality of muscle activation estimates are reduced and more sensitive to changes in joint angle (Staudenmann et al., 2006). Recently, it was shown that the problem of electrode alignment can be overcome with principal component analysis (PCA), which was applied on multiple monopolar EMG channels and is thus unbiased with respect to the muscle fiber direction (Staudenmann et al., 2006). So far, PCA-processed monopolar multi-channel EMG has been applied on the triceps brachii, and triceps surae (Staudenmann et al., 2006, 2009a), both mainly pennate and architecturally complex muscles (Chow et al., 2000; Kawakami et al., 1993). A substantial improvement in muscle activation estimates was achieved with 
PCA as compared to multiple bipolar EMG using the same electrodes (Staudenmann et al., 2006, 2009a), suggesting that the misalignment of (multiple) bipolar EMG is a cause of errors in the estimation.

Because the biceps brachii muscle (BB) has a mainly parallel fiber arrangement with the innervation zone midway of the longitudinal muscle direction (Aquilonius et al., 1984; Masuda et al., 1983), bipolar configurations can more easily be aligned to the muscle fiber direction. In line with this, many studies using EMG have shown motor unit potentials, starting at the middle of the muscle and propagating towards the distal and proximal ends of the BB fibers (Masuda et al., 1983; Merletti et al., 2003; Stalberg, 1966; Zwarts and Stegeman, 2003). Measuring propagating waves on both the proximal and the distal side of the innervation zone would in such ideal cases be redundant and would not add information on muscle activity and muscle force. However, heterogeneity in muscle activity in the medio-lateral, but also some in longitudinal direction within BB has been reported during voluntary contractions (ter Haar Romeny et al., 1984; van Zuylen et al., 1988).

The aim of this study was twofold. First, we investigated the degree of redundancy in EMG data obtained with multiple electrodes distributed over the parallel-fibered BB during an isometric elbow flexion (e.g. Staudenmann et al., 2009a). Different electrode configurations were tested (proximal-distal of the innervation zone, medio-lateral and longitudinal to muscle fibers) and the resulting muscle activation estimate was related to normalized elbow flexion force. Although the normalized flexion force is obviously determined by multiple synergistic and antagonistic muscles, BB is expected to be a major contributor (Kawakami et al., 1994). Hence we reasoned that any reduction in the difference between the EMG amplitude and the flexion force would reflect a better estimate of the contribution of the BB to the elbow moment, and thus a better estimate of activation of especially the $\mathrm{BB}$. On the one hand, we hypothesized that signals obtained from proximal and distal BB would contain redundant information, as they would reflect the same propagating waves and thus would not add to the quality of muscle activation estimates. Channels added in the medio-lateral direction over the two heads of BB, on the other hand, would provide additional information and hence improve estimates.

The second aim of this study was to test two processing methods: The aforementioned (multiple) bipolar configurations vs. processing the same monopolar channels with PCA. We hypothesized that PCA does not improve muscle activation estimates in BB, because the (multiple) bipolar EMG can be accurately aligned to the parallel muscle fibers. Some of the data have been presented previously in abstract form (Staudenmann et al., 2011).

\section{Methods}

\subsection{Subjects and procedures}

Eleven healthy men (mean \pm standard deviation: age, $27.3 \pm 8.3$ years; mass, $82.5 \pm 12.8 \mathrm{~kg}$; height, $1.8 \pm 0.7 \mathrm{~m}$ ) without neurological or cardiovascular disorders participated in the experiment. Informed, written consent was obtained before participation in the study. The Human Subjects Committee at the University of Colorado approved the protocol. Subjects were prepared for the experiment and seated in the setup as described previously (Staudenmann et al., 2009b). Briefly, before the experiment a maximum voluntary contraction (MVC) of elbow flexion was performed, which comprised a 2-3 s steady increase in force to MVC and a 23 s steady decrease to rest. MVCs were repeated three times and subjects were verbally encouraged. For the experiment, subjects had to generate an isometric (two-state isotonic) contraction, smoothly increasing from rest to an indicated target force of 50\%MVC in about $3 \mathrm{~s}$, holding it for about $4 \mathrm{~s}$, and again smoothly relaxing the contraction until rest again in about $3 \mathrm{~s}$ (see Fig. 1).

\subsection{Mechanical recording}

Subjects were seated in a chair with the left upper arm vertically aligned and the elbow flexed to a right angle with the forearm in the horizontal plane. The elbow was supported by a rounded surface that restrained movements of the arm. The forearm was in the neutral position (between supination-pronation) and tightly fixed in a wrist-hand-thumb orthosis (Orthomerica, Newport Beach, CA, USA) that was attached to a force transducer (Force-Moment Sensor, JR-3, Woodland, California, USA), to measure the upward force at the wrist. The position of the arm and forearm was aligned with the principal directions of the force transducer, such that elbow flexion mainly produced an upward force. The normalized flexion force was displayed on a screen $(1 \% \mathrm{MVC} / \mathrm{cm})$ in front of the subject and the force signal was recorded using a Power 1401 A-D converter (1000 samples/s, 12-bit resolution) and Spike2 software (Version 5.02, Cambridge Electronics Design, Cambridge, UK).

\subsection{Electrophysiological recording}

Multi-channel surface EMG was recorded from BB. We used adapted Soft-E Repositionable Cloth Series electrodes (Kendall, Boston, USA). The tissue around the metallic clipper connection was cut away in order to obtain small electrodes, which allowed us to generate the desired electrode resolution over the muscle. We attached $63 \pm 4$ electrodes over $\mathrm{BB}$, adapting to the muscle shape and size of the different subjects. The first electrode was placed over the intersection of the innervation zone (halfway between proximal and distal edges of BB (Masuda et al., 1983)) and the septum between the BB muscle heads, which was determined by palpation (Fig. 1). Then electrodes were added in medio-lateral direction with an inter-electrode distance of $\pm 1.5 \mathrm{~cm}$ until the medial and lateral edges of BB were reached. Parallel to the first row over the innervation zone, we added proximal and distal rows of electrodes with the same inter-electrode distance, resulting in a homogenous electrode coverage over the entire BB (Fig. 1). The reference electrode was placed over the lateral epicondyle of the elbow and the electrodes were connected to the amplifier (REFA, TMSi, Oldenzaal, NL), which collected monopolar EMG signals (input impedance $>10^{12} \Omega$, AD converted at 2000 samples/s, 24-bits resolution, effective low-pass frequency $480 \mathrm{~Hz}$ avoiding aliasing).

\subsection{Data analysis}

The data were analyzed with Matlab (The Mathworks, Inc., Natick, MA, USA). Force signals were up-sampled (using cubic spline interpolation) and aligned to the EMG signals with the synchronization pulse. The plateau phase was determined based on the force pattern reaching and sustaining the desired 50\%-MVC.

Monopolar EMG signals were first high-pass filtered $(10 \mathrm{~Hz}$, Butterworth, 1st order, bi-directional). Subsequently, the following four electrode configurations were constructed, using electrode pairs in longitudinal muscle direction (see Fig. 1): proximal or distal single bipolar channels over BB (1p, 1d), single proximal and distal rows of multiple bipolar channels $(2 \mathrm{p}, 2 \mathrm{~d})$, multiple rows of bipolar channels over the entire proximal or distal part (3p, 3d), and multiple rows of bipolar channels over the entire BB (4). Alternatively, the same monopolar channels, which were used for the different configurations described above, were processed with 

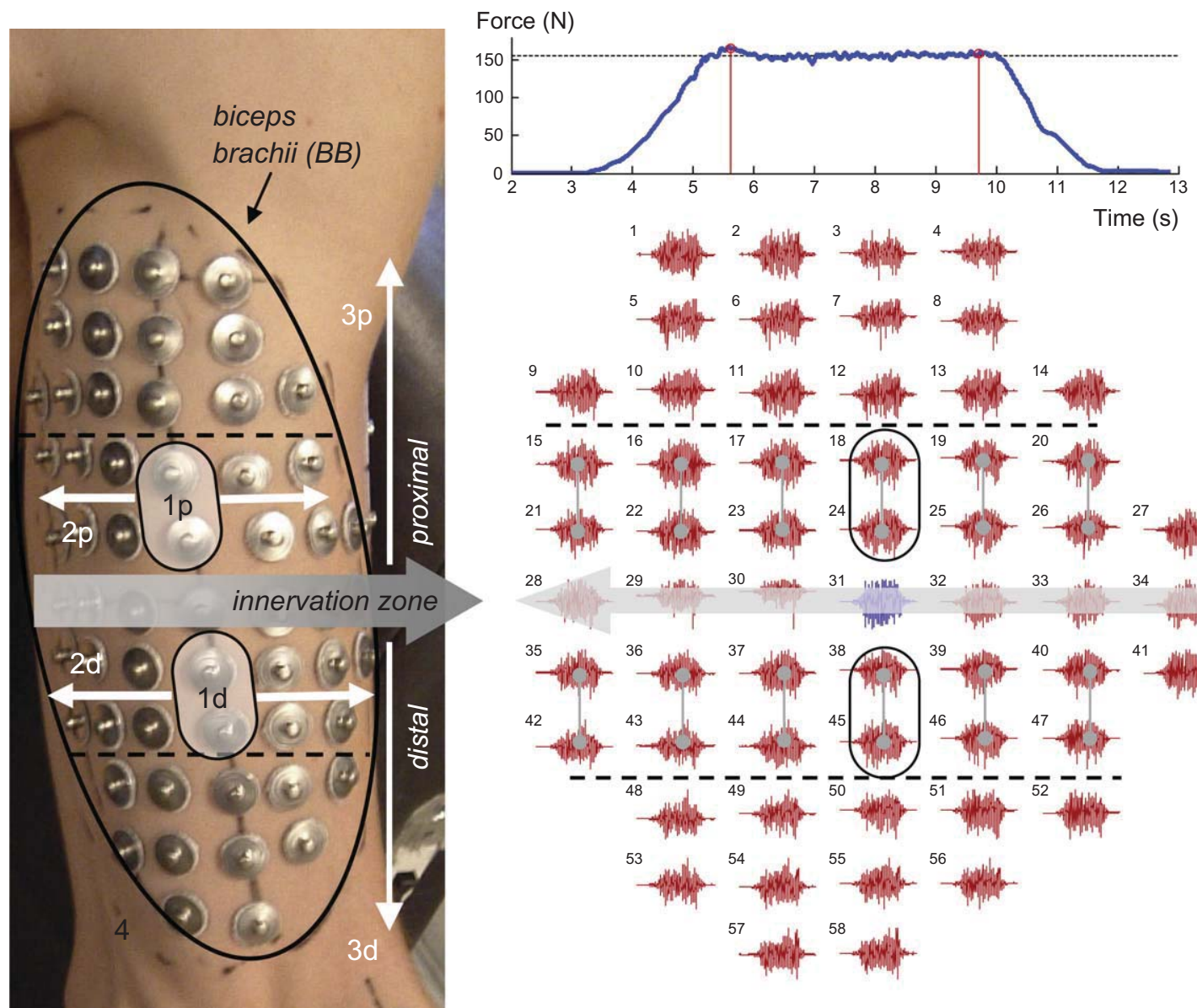

Fig. 1. One representative subject showing the electrode coverage over BB. On the right upper panel the force profile of the isometric contraction. The dashed line shows the target force ( $150 \mathrm{~N}$ for this subject), which had to be reached and held for about $4 \mathrm{~s}$ (vertical stem lines). Below the high-pass filtered 58 monopolar EMG signals over BB for this particular subject. All electrode configurations (1-4) consisted of (multiple) neighboring bipolar channels in longitudinal muscle direction: Proximal or distal single bipolar channel (1p, 1d; indicated by the ellipses), proximal or distal single rows of multiple longitudinal bipolar channel (2p, 2d; indicated by the dot-line connections), in the same way multiple overlapping rows of bipolar channel over the entire proximal or distal part (3p, 3d), and multiple overlapping rows of bipolar channels over the entire $\mathrm{BB}(4)$.

PCA as proposed in a previous study (Staudenmann et al., 2006), yielding the conditions: 2pPCA, 2dPCA, 3pPCA, and 3dPCA. The first four principal components were discarded, as a means to eliminate common information from the monopolar EMG data in an unbiased way (Staudenmann et al., 2006, 2009a).

The signals of the channels used in the configurations were rectified, averaged, low-pass filtered $(2 \mathrm{~Hz}$, Butterworth, $1 \mathrm{st}$ order, bidirectional), and both, EMG and force were normalized over the average plateau phase. As a measure of quality of the estimated level of muscle activation we used the root mean square difference (RMSD) between normalized EMG and normalized force for the entire block-shaped profiles as is exemplified in Fig. 2. Finally, we determined to what extent independent information was present between proximal or distal parts of BB. We used correlation coefficients between the distal and proximal EMG amplitudes for electrode configurations 1-3 and for the two processing methods (bipolar vs. PCA).

\subsection{Statistics}

The results were statistically tested with repeated measures analyses of variance (ANOVA; SPSS 19, Inc., Chicago, IL, USA) with significance at $5 \%$. First, we tested for differences in RMSD between configurations (1-4) and locations (proximal vs. distal). Post-hoc pairwise ANOVA's were used to test for differences between

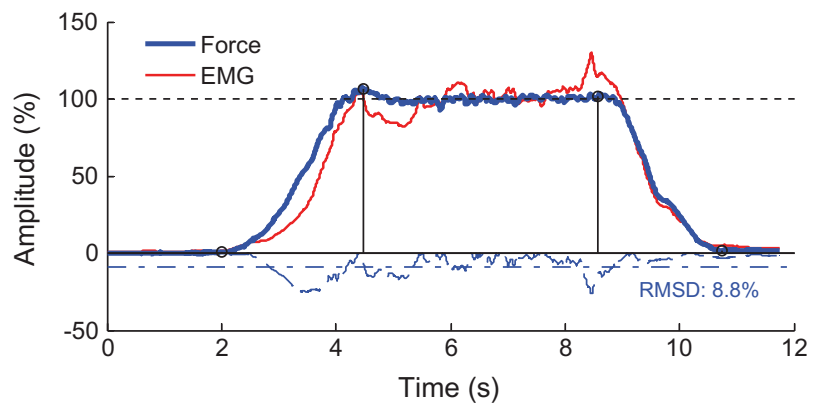

Fig. 2. The association between force and EMG amplitude for one representative subject (configuration 4). The dashed line at $100 \%$ shows the target force level as indicated to the subjects. Vertical lines indicate the force plateau phase. The curve below zero indicates the Euclidean absolute difference between force and EMG. The stripped line below zero indicates the root mean square difference (RMSD) over the entire pattern $(\sim 0-12 \mathrm{~s})$ between force and EMG. Note that a low RMSD represents a high match between force and EMG.

specific configurations or locations. Then, we tested for differences in RMSD between processing methods (bipolar vs. PCA). In addition, we compared correlation coefficients (after Fisher-z transformation) with configuration as the independent factor, and twoway ANOVA was used with configuration and processing method as independent factors. 


\section{Results}

During the 50\%-MVC contractions, subjects produced a flexion force measured at the wrist of $150.2 \pm 25.1 \mathrm{~N}$ (mean \pm standard deviation; elbow moment: $42.3 \pm 7.3 \mathrm{Nm}$ ). The overall comparison of the configurations (1-4) showed a significant effect on the RMSD $(p<0.001$; Fig. 3$)$. A post hoc comparison yielded no significant differences ( $p=0.098$ ) between proximal vs. distal single bipolar configurations ( $1 \mathrm{p}$ vs. $1 \mathrm{~d}$ ), whereas distal configurations ( $2 \mathrm{~d}$ and $3 \mathrm{~d}$ ) yielded significantly lower RMSD values (up to $8 \%, p=0.039$ and $p=0.027$, respectively) than corresponding proximal configurations ( $2 \mathrm{p}$ and $3 \mathrm{p})$. For the proximal locations, configurations ( $1 \mathrm{p}$, $2 p, 3 p)$ were not significantly different. For the distal locations, configuration $1 d$ yielded a higher RMSD than $2 d$ and $3 d$ ( $p=0.004$ and $p=0.001$ respectively). The latter two were not significantly different from each other. Finally, the last two configurations ( $3 \mathrm{~d}$ vs. 4) showed no significant difference.

The contribution of the first principal component to the variance of all monopolar channels was $77.8 \pm 8.7 \%$ and the sum of the selected components (5th to last principal component) corresponded to $9.1 \pm 5.3 \%$ of the total monopolar power. As can be seen in Fig. 4, PCA-processed EMGs yielded a substantial (12-22\%) and significant reduction in RMSD compared to the bipolar configurations based on the same monopolar channels $(p=0.001)$. Differences between configurations and locations were similar for PCA-processed data as for the bipolar configurations, with a significantly lower RMSD for distal as compared to proximal configura-

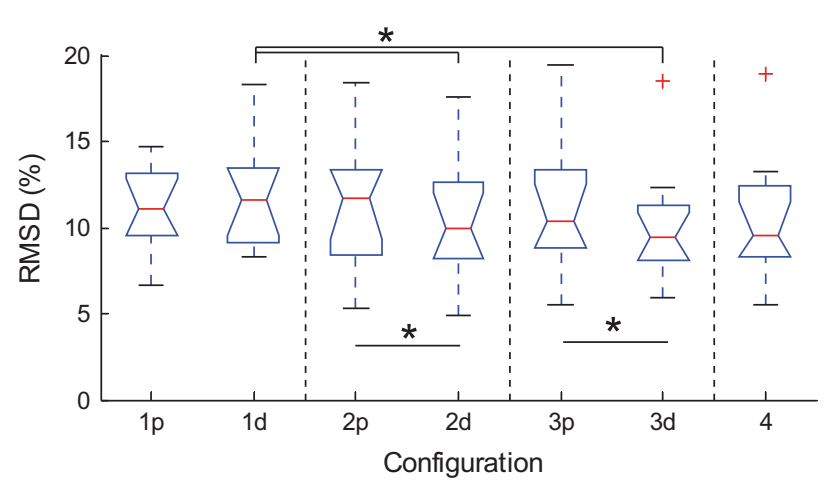

Fig. 3. Overall result of the quality of EMG-based force estimates as quantified by RMSD. The numbers 1-4 correspond to the (multiple) bipolar configurations as shown in Fig. 1. The middle line of the boxplot represents the median value, error bars the range and lower/higher boxes with notches represent the interquartile range and plus signs show outliers. Note the significantly $\left(^{*}\right)$ lower RMSD for distal as compared to proximal configuration, for conditions 2,3 .
Table 1

Average improvements (\%-RMSD) of muscle activation estimates of the configurations (2-4) as compared to the a conventional single bipolar configuration (1, see Fig. 1).

\begin{tabular}{|c|c|c|c|c|c|}
\hline $2 p$ & $2 p P C A$ & $3 p$ & $3 p P C A$ & 4 & $4 P C A$ \\
\hline $\begin{array}{l}\text { Proximal } \\
5 \%\end{array}$ & $16 \%$ & $6 \%$ & $22 \%$ & Entire $B B$ & \\
\hline $\begin{array}{l}\text { Distal } \\
12 \%\end{array}$ & $30 \%$ & $14 \%$ & $32 \%$ & $11 \%$ & $34 \%$ \\
\hline
\end{tabular}

tions ( $p=0.001)$, and no significant difference between 2PCA and $3 P C A(p>0.083)$, neither between 3dPCA and 4PCA $(p=0.556)$.

Compared to the single bipolar EMG, the multiple bipolar configurations yielded 5-16\% reductions in RMSD and the PCA-processed EMG yielded $16-34 \%$ reductions in RMSD (Table 1).

EMG amplitudes of proximal and distal configurations (1-3) over BB showed very high correlations over the entire trapezoidal force pattern $(r=0.99 \pm 0.01)$. To obtain a more sensitive measure, we additionally investigated the correlation over the plateau phase only. Significant differences in correlations were found between the first three configurations and between processing methods $(p<0.001$, Fig. 5). The lowest correlation was found between the single bipolar channels (1p vs. 1d). No significant difference in correlations was found between configurations 2 and 3 across processing methods $(p=0.882$ ), whereas PCA-processed signals had

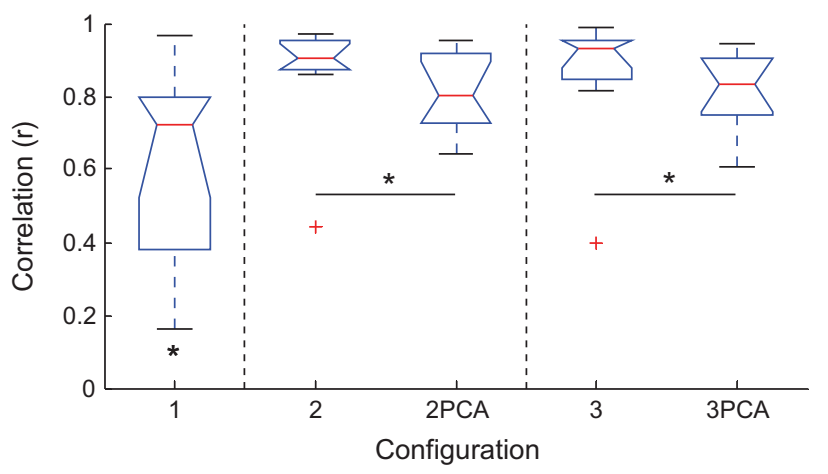

Fig. 5. Correlation between proximal and distal EMG activities of BB for the first three electrode configurations. The numbers $1-3$ corresponds to the configurations as shown in Fig. 1. The middle line of the boxplot represents the median value, error bars the range and lower/higher boxes with notches represent the interquartile range and plus signs show outliers. Note the lowest correlation between proximal and distal using single pair bipolar configuration and the lower correlation for PCAprocessed EMG.

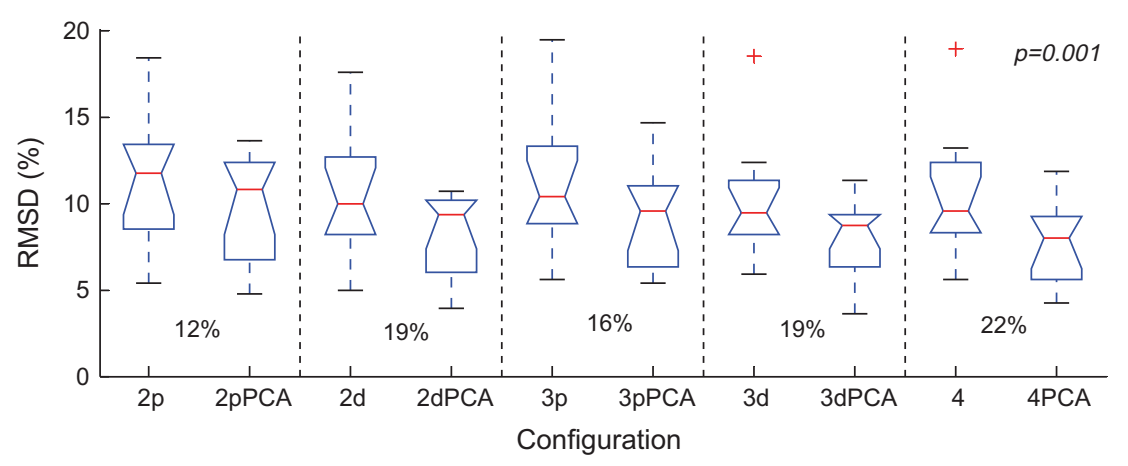

Fig. 4. Overall results showing the quality of EMG-based force estimates (RMSD) for processing method (bipolar vs. PCA). The numbers $1-4$ corresponds to the configurations as described in Fig. 1. The middle line of the boxplot represents the median value, error bars the range and lower/higher boxes with notches represent the interquartile range and plus signs show outliers. PCA-processed EMG showed a significant and considerable improvement $(p=0.001)$ in RMSD as compared to (multiple) bipolar EMG, when using the monopolar input channels. 
significantly lower correlations $(6 \%, p=0.006)$ across proximal and distal locations as compared to the bipolar configurations.

\section{Discussions}

The first aim of this study was to analyze to which extent the EMG obtained over different parts of the BB during an isometric arm flexion is redundant. Therefore four different electrode configurations were tested with regard to the quality of muscle activation estimates. The second aim was to compare two different processing methods (bipolar vs. PCA) using the same monopolar input channels. BB is an ideal muscle to analyze the degree of redundancy within a muscle in more detail, since bipolar configurations can be aligned with the muscle fiber direction and over the muscle parts proximal and distal of the innervation zone. We chose an isometric, two-state isotonic contraction, in order to be independent of non-linear relationships between EMG and force (e.g. Hof and van den Berg, 1977; Lawrence and De Luca, 1983). Moreover this simple contraction type is expected to generate a more homogeneous behavior between synergists (e.g. van Zuylen et al., 1988) (e.g. brachioradialis) which will thus increase the association between BB activation and the elbow moment, because the contribution of synergists can expected to be stationary.

\subsection{Redundancy or heterogeneity within $B B$}

The lack of a difference in RMSD between bipolar configurations (1 and 2; Fig. 1), at first sight suggests redundancy of additional channels in the medio-lateral direction of BB. However, when processing the same monopolar channels of configuration 2 with PCA a 16\% lower RMSD was found as compared to configuration 1, indicating that activity is heterogeneously distributed over the mediolateral direction of BB.

When using all proximal or all distal channels, independent of the processing method (bipolar vs. PCA), RMSD was not significantly lower compared to one medio-lateral row of electrodes (configurations 3 vs. 2). Thus, information obtained by placing additional channels in the longitudinal direction appeared largely redundant for both proximal and distal portions of BB. This finding can be explained based on propagation of waves as the only difference between longitudinal channels in BB (Masuda and Sadoyama, 1986), which would obviously not add information on muscle activation estimates. The single bipolar EMG showed a high variance of the correlation between the proximal and distal part of BB (Fig. 5), which indicates heterogeneity between proximal and distal parts of BB in some subjects. However, since configurations with multiple channels (configurations 2 and 3 ) yielded substantially higher correlations, this is more likely due to the imprecise estimates of muscle activation obtained with a single bipolar configuration (Farina et al., 2004; Staudenmann et al., 2005).

\subsection{Distal vs. proximal configurations}

For all configurations, except the single bipolar, distal channels showed a systematically lower RMSD as compared to the proximal electrodes ( $\sim 7 \%$ for bipolar and $\sim 12 \%$ for PCA). As shown in Fig. 5 , EMG amplitudes of proximal and distal parts of $\mathrm{BB}$ were more strongly correlated for multiple bipolar configurations than for PCA $(r=0.87, r=0.81$, respectively). These results indicate that the multiple bipolar configurations captures more homogenous patterns, most likely dominated by information from propagating waves, which is in line with previous studies (Masuda and Sadoyama, 1986). PCA-processed muscle activation estimates also showed relatively high correlations, but appeared to extract more independent information when relating proximal to distal parts.
Since the RMSD obtained with all electrodes over the whole BB was not different from the distal configurations only, we conclude that redundancy is present between proximal and distal parts of $\mathrm{BB}$. However, the brachialis muscle, a synergist of the BB in elbow flexion, arises from a broad origin about midway of the humerus and inserts into the ulna (Herbert and Gandevia, 1995). Due to the fusiform shape of the distal part of BB (e.g. Pappas et al., 2002), the largest distance between electrodes and brachialis will be over the central portion of $\mathrm{BB}$, whereas co-lateral electrodes will be closer to the larger anatomical organization of brachialis muscle. In addition, BB and brachialis are not purely co-activated during voluntary contractions (Naito, 2004). Contributions of the brachialis muscle to both, the measured force and the distally measured EMG may thus be responsible for the systematically lower RMSD of the configurations over the distal part of BB (cf. Naito, 2004). The larger difference in RMSD between proximal and distal configurations with PCA than with the bipolar EMG, suggests that PCA processing is more representative of the motor units within the muscle, probably also of deeper motor units (e.g. Staudenmann et al., 2010).

\subsection{Improvement in muscle activation estimates with $P C A$}

For all four configurations tested, PCA-processed monopolar EMG showed substantial improvements in muscle activation estimates (12-22\%, Fig. 4) as compared to (multiple) bipolar EMG. We did not expect this substantial improvement with PCA over a parallel-fibered muscle such as BB, because bipolar electrodes can be aligned to the fiber direction. When discarding the first components of monopolar EMG signals with PCA, this acts similarly to a bipolar configuration, which per definition also discards common information, but PCA is unbiased in respect to the muscle fiber direction (Staudenmann et al., 2006, 2010). However, BB may have a more complex architecture than commonly assumed with an overall fusiform shape and obliquely oriented fibers at the distal portion of BB (Pappas et al., 2002). This would cause some misalignment of distal bipolar EMG with respect to the muscle fiber direction, which has been shown to reduce the quality of EMGbased force estimates (Staudenmann et al., 2006). However, because PCA still showed substantial improvements, also over the proximal part of BB (12-16\%), the misalignment between bipolar EMG and fiber direction appears not the main cause for the substantial improvements with PCA. Another aspect, as already mentioned above, is that PCA may be more representative of all active motor units in the muscle (cf. Staudenmann et al., 2010). Accordingly, not only BB can be better represented in PCA-processed EMG, but also information form brachialis is expected to be better represented over the distal portion of BB as using a bipolar EMG. It remains to be shown how PCA succeeds in optimizing signal extraction in muscle activation estimates of parallel-fibered muscles.

\subsection{Limitations}

When measuring the flexion force at the wrist, we only have information on the net elbow moment. The obviously not perfect match between EMG and force (Fig. 2) might to a part be due to the mechanical output of the remaining muscles crossing the elbow (Buchanan et al., 1989). However, when force estimates of the BB improve, this should yield a closer correspondence with the total elbow flexion force as well, given the large contribution of BB to elbow flexion force (Kawakami et al., 1994). It should be noted that in all tests we compare the same data with only the BB EMG data processed differently, this means that when the activation of synergists or antagonists fluctuates during the trial, this 
lowers the correlation between EMG and force, but it does not bias our comparisons.

We used comparisons of quality of muscle activation estimates and the correlations between EMG channels obtained with various electrode locations, configurations and processing methods to determine to what extent activation of the BB is heterogeneous. It should be noted that insofar these comparisons involve differences in numbers of electrodes used in obtaining an estimate of muscle activity, positive results could partly be caused by the improvement of the signal-to-noise ratio obtained by adding channels with very similar signals, distorted by independent instrumentation noise, as predicted by the conventional averaging theorem. However, this bias appears limited, as is illustrated by the lack of difference in RMSD between configurations 1 ( 2 channels) and 2 ( $\sim 12$ channels). This probably indicates that instrumentation noise levels in the measured signals were too low to exert substantial influence on the RMSD.

Crosstalk can be a problem with surface EMG. Co-lateral electrodes over BB are closer to neighboring muscles and could contain some crosstalk. We expect, however, minimal disturbance of signals originating for instance from triceps brachii because of the anatomical structure of the upper arm most of the electrodes were above the muscle of interest (BB and brachialis). It has to be realized, however, that the representativeness of the active (heterogeneous) motor unit potentials in the surface EMG is even more important than problems of crosstalk (e.g. Staudenmann et al., 2010).

Non-conventional processing methods of a single bipolar EMG (e.g. whitening) can also lead to an improvement of signal extraction (Clancy and Hogan, 1994; Hashemi et al., 2012; Staudenmann et al., 2007). In this study we were interested to see the gain in the quality of muscle activation when comparing PCA-processed monopolar EMG to conventionally processed single bipolar EMG.

\section{Conclusion}

The use of multiple bipolar EMG channels can substantially improve the quality of muscle activation estimates compared to a single bipolar channel, also in a parallel-fibered muscle such as BB. Within $\mathrm{BB}$, heterogeneous activity was found mainly in the medio-lateral direction, whereas additional signals from multiple longitudinally arranged electrodes appeared mainly redundant. Activity obtained over the distal BB corresponded more closely with elbow flexion force than activity over the proximal part, most likely because activity of brachialis was also captured by the distal electrodes. Over all configurations tested, PCA-processed EMG yielded more accurate estimates of muscle activation than multiple bipolar EMG using the same monopolar channels (12-22\%), thus the outcome depends on the processing method. The misalignment between bipolar EMG to the muscle fibers is expected to have little effect for the improvement obtained with PCA, but another factor, yet to be identified, must contribute to the improvement in the quality of muscle activation estimates.

\section{Conflict of interest}

None of the authors had any financial or personal conflict of interest with regard to this study.

\section{Acknowledgments}

The data collection was supported by an award (No. 115183) from the Swiss National Science Foundation (to D. Staudenmann) for a post-doctoral fellowship at the Neurophysiology of Movement Laboratory (Prof. R.M. Enoka), University of Colorado, USA.

\section{References}

Aquilonius SM, Askmark H, Gillberg PG, Nandedkar S, Olsson Y, Stalberg E. Topographical localization of motor endplates in cryosections of whole human muscles. Muscle Nerve 1984;7:287-93.

Bojson-Moller J, Hansen P, Aagaard P, Svantesson U, Kjaer M, Magnusson SP. Differential displacement of the human soleus and medial gastrocnemius aponeuroses during isometric plantar flexor contractions in vivo. J Appl Physiol 2004;97:1908-14.

Buchanan TS, Rovai GP, Rymer WZ. Strategies for muscle activation during isometric torque generation at the human elbow. J Neurophysiol 1989;62:1201-12.

Chow RS, Medri MK, Martin DC, Leekam RN, Agur AM, McKee NH. Sonographic studies of human soleus and gastrocnemius muscle architecture: gender variability. Eur J Appl Physiol 2000;82:236-44.

Clancy EA, Hogan N. Single site electromyograph amplitude estimation. IEEE Trans Biomed Eng 1994;41:159-67.

Darby J, Hodson-Tole EF, Costen N, Loram ID. Automated regional analysis of Bmode ultrasound images of skeletal muscle movement. J Appl Physiol 2012;112:313-27.

De Luca CJ. The use of surface electromyography in biomechanics. J Appl Biomech 1997; 13:135-63.

Eriksson PO, Stalberg E, Antoni L. Flexibility in motor-unit firing pattern in the human temporal and masseter muscles related to type of activation and location. Arch Oral Biol 1984;29:707-12.

Farina D, Merletti R, Enoka RM. The extraction of neural strategies from the surface EMG. J Appl Physiol 2004;96:1486-95.

Finni T, Hodgson JA, Lai AM, Edgerton VR, Sinha S. Nonuniform strain of human soleus aponeurosis-tendon complex during submaximal voluntary contractions in vivo. J Appl Physiol 2003;95:829-37.

Hashemi J, Morin E, Mousavi P, Mountjoy K, Hashtrudi-Zaad K. EMG-force modeling using parallel cascade identification. J Electromyogr Kinesiol 2012;22:469-77.

Herbert RD, Gandevia SC. Changes in pennation with joint angle and muscle torque: in vivo measurements in human brachialis muscle. J Physiol 1995;484:523-32.

Hermens HJ, Freriks B, Disselhorst-Klug C, Rau G. Development of recommendations for SEMG sensors and sensor placement procedures. J Electromyogr Kinesiol 2000;10:361-74.

Hof AL, van den Berg J. Linearity between the weighted sum of the EMGs of the human triceps surae and the total torque. J Biomech 1977;10:529-39.

Holtermann A, Roeleveld K, Karlsson JS. Inhomogeneities in muscle activation reveal motor unit recruitment. J Electromyogr Kinesiol 2005;15:131-7.

Inman VT, Ralston HJ. Relation of human electromyogram to muscular tension. EEG Clin Neurophysiol 1952;4:187-94.

Kawakami Y, Abe T, Fukunaga T. Muscle-fiber pennation angles are greater in hypertrophied than in normal muscles. J Appl Physiol 1993;74:2740-4.

Kawakami Y, Nakazawa K, Fujimoto T, Nozaki D, Miyashita M, Fukunaga T. Specific tension of elbow flexor and extensor muscles based on magnetic resonance imaging. Eur J Appl Physiol Occup Physiol 1994;68:139-47.

Lawrence JH, De Luca CJ. Myoelectric signal versus force relationship in different human muscles. J Appl Physiol 1983;54:1653-9.

Masuda T, Sadoyama T. The propagation of single motor unit action potentials detected by a surface electrode array. Electroencephalogr Clin Neurophysiol 1986;63:590-8.

Masuda T, Miyano H, Sadoyama T. The distribution of myoneural junctions in the biceps brachii investigated by surface electromyography. Electroencephalogr Clin Neurophysiol 1983;56:597-603.

Merletti R, Lo Conte L, Avignone E, Guglielminotti P. Modeling of surface myoelectric signals - Part I: model implementation. IEEE Trans Biomed Eng 1999;46:810-20.

Merletti R, Farina D, Gazzoni M. The linear electrode array: a useful tool with many applications. J Electromyogr Kinesiol 2003;13:37-47.

Naito A. Electrophysiological studies of muscles in the human upper limb: the biceps brachii. Anat Sci Int 2004;79:11-20.

Pappas GP, Asakawa DS, Delp SL, Zajac FE, Drace JE. Nonuniform shortening in the biceps brachii during elbow flexion. J Appl Physiol 2002;92:2381-9.

Stalberg E. Propagation velocity in human muscle fibers in situ. Acta Physiol Scand Suppl 1966;287:1-112.

Staudenmann D, Kingma I, Stegeman DF, van Dieen JH. Towards optimal multichannel EMG electrode configurations in muscle force estimation: a high density EMG study. J Electromyogr Kinesiol 2005;15:1-11.

Staudenmann D, Kingma I, Daffertshofer A, Stegeman DF, van Dieen JH. Improving EMG-based muscle force estimation by using a high-density EMG grid and principal component analysis. IEEE Trans Biomed Eng 2006;53:712-9.

Staudenmann D, Potvin JR, Kingma I, Stegeman DF, van Dieen JH. Effects of EMG processing on biomechanical models of muscle joint systems: sensitivity of trunk muscle moments, spinal forces, and stability. J Biomech 2007;40:900-9.

Staudenmann D, Kingma I, Daffertshofer A, Stegeman DF, van Dieen JH. Heterogeneity of muscle activation in relation to force direction: a multichannel surface electromyography study on the triceps surae muscle. J Electromyogr Kinesiol 2009a;19:882-95.

Staudenmann D, Rudroff T, Enoka RM. Pronation-supination torque and associated electromyographic activity varies during a sustained elbow flexor contraction but does not influence the time to task failure. Muscle Nerve 2009b;40:231-9.

Staudenmann D, Roeleveld K, Stegeman DF, van Dieen JH. Methodological aspects of SEMG recordings for force estimation - a tutorial and review. J Electromyogr Kinesiol 2010;20:375-87. 
Staudenmann D, Stegeman DF, van Dieen JH. EMG-based muscle force estimation with multi-channel electrodes on arm flexor muscles. Brussels, ISB2011; 2011.

ter Haar Romeny BM, van der Gon JJ, Gielen CC. Relation between location of a motor unit in the human biceps brachii and its critical firing levels for different tasks. Exp Neurol 1984;85:631-50.

van Zuylen EJ, Gielen CC, Denier van der Gon JJ. Coordination and inhomogeneous activation of human arm muscles during isometric torques. J Neurophysiol 1988;60:1523-48.

Vieira TM, Loram ID, Muceli S, Merletti R, Farina D. Postural activation of the human medial gastrocnemius muscle: are the muscle units spatially localised? J Physiol 2011;589:431-43.

Wolf SL, Ammerman J, Jann B. Organization of responses in human lateral gastrocnemius muscle to specified body perturbations. J Electromyogr Kinesiol 1998;8:11-21.

Zwarts MJ, Stegeman DF. Multichannel surface EMG: basic aspects and clinical utility. Muscle Nerve 2003;28:1-17. 